\title{
Animal models of chronic pain. Are naturally occurring diseases a potential model for translational research?
}

\author{
Daniel E. Herzberg, Hedie A. Bustamante*
}

\begin{abstract}
Despite the vast amount of molecular data obtained from classical pain studies, there is an ongoing translational pain model crisis reflected by the reduced amount of new effective and safe compounds developed to treat chronic pain in humans. Naturally occurring chronic pain in animals may offer some advantages over induced models of chronic pain, including a natural development of the condition that induces pain, the heterogenicity of the population that affects, and the chronologic age in which they develop, among others. The identification and study of naturally occurring painful diseases that resemble a particular chronic painful condition in humans has been proposed as a potential tool to investigate the molecular mechanisms and thus, accelerating drug development at the preclinical and clinical level. Currently, certain types of chronic pain in companion and large animals have gained attention as potential translational models of chronic pain. Examples of these include canine and feline osteoarthritis, neoplastic diseases as osteosarcoma and bovine and equine lameness. The present review describes the limitations of animal models of chronic pain and briefly enters in how naturally occurring pain models could represent a translational approach to chronic pain.

Key words: chronic pain, animal models, translational.
\end{abstract}

\section{INTRODUCTION}

Chronic pain (CP) develops from multiple clinical conditions defined by longstanding pain that adversely impacts the quality of life (Gereau IV et al 2014), affecting at least 20-30\% of the general population and individuals of all ages, races and genders. The International Association for the Study of Pain (IASP) defines chronic pain as "pain that lasts or recurs for more than three months" (Treede et al 2015). This definition has evolved in the last few years from a unidimensional to a multidimensional state, including sensitive, cognitive and emotional components (Kumar and Elavarasi 2016). Chronic pain has many special features, but one of the most prominent originates from a complex dysfunction of the nervous system and lasts after the normal healing period. It is paroxistic and it lacks any adaptative function, associated to biochemical and phenotypical changes in the nociceptive pathway (Ueda 2008). Interestingly, the global burden of pain is large and growing. The IASP estimates that 1 in 5 patients experience pain and that 1 in 10 patients are diagnosed with CP yearly, with chronic pain being the most common cause for treating medical care (Enright and Goucke 2016). Moreover, there is agreement that in low- and middle-income countries $\mathrm{CP}$ is poorly managed with more than $80 \%$ of patients not receiving proper treatment (Jackson et al 2016). Finally, during 2019, the IASP published the classification of chronic pain for the International Classification of Diseases (ICD-11), which will improve recognition and diagnosis

Received: 16.10.2019.

Accepted: 11.05.2020.

Instituto de Ciencias Clínicas Veterinarias, Facultad de Ciencias Veterinarias, Universidad Austral de Chile, Valdivia, Chile.

*Corresponding author: H Bustamante; hbustamante@uach.cl of different clinical conditions, thus establishing chronic pain as a health condition (Treede et al 2019).

The transition between acute and chronic pain is another key component in the pain paradigm. Woolf et al (2010) mentioned that the physiopathological differences between acute and chronic must be thoroughly defined before developing potential new treatments. Recently, concepts as "adaptative" and "non-adaptative" have been described and used in order to obtain a better classification of chronic pain (Adrian et al 2017). The development of a "non-adaptative" pain is always related to neuronal and glial plasticity that leads to central sensitization and maintains the painful sensation after the healing period (Woolf 2011). This plasticity in the Central Nervous System (SNC) has also been associated with clinical features of pain, including the exaggerated pain response after a painful stimuli (Hyperalgesia); and the painful response after a non-painful stimuli (Allodynia). Furthermore, chronic pain has been classified according to its origin in nociceptive, neuropathic and nociplastic.

Nociceptive pain includes inflammatory conditions such as osteoarthritis and it can be defined as "pain that arises from actual or threatened damage to non-neuronal tissue and is due to the activation of nociceptors". Neuropathic pain includes conditions such as diabetic neuropathy, chemotherapy induced neuropathy, phantom limb pain and it can be defined as "pain caused by a lesion or disease of the somatosensory system". Nociplastic pain was recently introduced by the IASP Council and includes fibromyalgia. Nociplastic pain can be defined as "pain that arises from altered nociception despite no clear evidence of actual or threatened tissue damage causing the activation of peripheral nociceptors or evidence for disease or lesion of the somatosensory system causing pain". Although most of the painful conditions mentioned earlier have been studied in human patients, they commonly occur in 
animals. This finding has led to a new area of research in which naturally occurring diseases could confirm and/or elucidate previously confirmed mechanisms in rodents. The present review describes the limitations of animal models of chronic pain and briefly enters in how naturally occurring pain models could represent a translational approach to chronic pain.

\section{EXPERIMENTAL PAIN MODELS}

A continuous increase in the used of animal models to study biological processes and disease has been noted since the early 1900s (Mogil 2009) and pain transmission and abnormal pain processing has been the focus for research since the late 19th century. Moreover, the study of pain has extensively relied on preclinical animal models in order to determine the sensory and psychological complexities of this condition (Burma et al 2017). Animal models of pain have been mainly developed in order to recreate a pathological painful condition aiming to identified a mechanistic explanation of pain biology and the identification of therapeutic targets to develop new compounds to safely alleviate pain (Gregory et al 2013).

In order to obtain accurate data that can be effectively translated to humans, an animal model of pain must encompass a sufficient high face validity (the capacity of the model to recreate all possible signs and symptoms of the disease that supposed to model) and predictive validity (the capacity of a model to be sensitive to an analgesic compound and insensitive to a non-analgesic compound) (Mogil 2009). Nonetheless, in recent years it has become clear that the face and predictive validity of current pain models is not sufficient to fully complete the task they were implemented for originally (Mao 2009, Vierck et al 2008, Quessy 2010, Clark 2016). Similarly, the credibility of efficacy data obtained from animal models of pain has been questioned (Mogil et al 2010).

Although animal models of pain have proven successful in elucidating pathophysiological mechanisms of chronic pain, the impact of these models in drug development has been disappointing, with failure rates in the clinical phase of around 90 to $95 \%$ (Arrowsmith 2012). In 2014, the reported likelihood of approval of an experimental analgesic drug to pass to Phase I trial was only $10.7 \%$ (Hay et al 2014). This report remarks the fact that no novel analgesics compounds have been developed since the 70's to safely and effectively treat chronic pain.

Several strategies have been proposed in order to improve this "translational gap" between scientific data and new compound development. These strategies include the higher refinement of the current models, the development of new and more accurate models, the replacement of evoked measure behaviour for operant behaviour measurement, and least but not last to include the measurement of variables that better reflect "quality of life" (Blackburn-Munro 2004, Mogil 2009, Quessy 2010, Clark 2016). It is important to consider that the limitations here described by any means overshadow the discoveries and advances made from these models, which according to Burma et al (2017) have been instrumental in advancing our understanding of the mechanistic under-pinning's of pain states and developing and testing new analgesic compounds.

According to Mogil (2009), an animal pain model must encompass three components: a subject, an assay and a measured outcome.

\section{THE SUBJECTS}

Many animal species, including laboratory animals such as rats, mouse, rabbits, small companion animals, large animals, and exotic species have been used to study the underlying mechanisms of chronic pain. During the '60's and 70's, mouse, rats, dogs, cats, and rabbits were the most used animals for pain studies. With the arrival of a new century, the development of transgenic mice which were incorporated in pain research (Mogil 2009). However, there are some behavioural inconvenients in mouse, such as difficulties in conditioning and other technicisms that make rats continue in the first place for pain studies (Wilson and Mogil 2001) and to the present-day rats have been employed in the vast majority of pain studies. Nonetheless, a recent search in the Pubmed database using the terms "pain"/"chronic pain" and "rat"/"mice" revealed that during 2019 a total of 1,590 papers used rats and 1,559 papers used mice as experimental models. Regarding chronic pain, 526 and 467 papers described the used of rats and mice, respectively. The highly extensive use of rodents for pain research is based mainly in the similarities in the neuroanatomy and physiology across mammalian species (Burma et al 2017). In order to reduce response variability, rodents employed in pain studies are frequently restricted to a certain breed, young age and one sex. Nonetheless, chronic pain states in humans are more frequent to occur in median-age or older, mainly women patients. Based on this, several authors have argued that restricting experimental animals to a certain age and gender do not represent the whole population to which the painful condition is modelled (Klinck et al 2017). Growing evidence has shown age-dependent differences in behavioural pain responses (Weyer et al 2016). Also, sexual dimorphism has an enormous impact on the development of chronic pain (Burma et al 2017).

\section{THE ASSAYS}

Several assays have been developed over the years, as pain is not a unitary phenomenon (Mogil 2009). Most assays offer the possibility to explore the pain system under controlled settings (Sunil kumar Reddy et al 2012). Similarly, assays have been classified in different manners. Walker et al (1999), classified the assays as somatic (acute nociceptive and pathological) and visceral. According 
to Mogil (2018), chronic neuropathic pain assays were developed as a response to the short duration of pain that the traditional models originated at that time. Furthermore, Sunil kumar Reddy et al (2012) mentioned that, mechanistically, the most important categories should include both peripheral and central sensitization, since both of them occur during pain.

Recently, Mogil (2018) described different assays for pain evaluation, which are summarised in figure 1 . This description classifies the existing assays according to the most common features described over the years. For the purpose of this review, we will briefly describe the most common assays of tonic pain and neuropathic pain.

\section{ASSAYS OF TONIC PAIN}

Inflammatory pain models used different irritants injected into the skin, paw, muscle, joint, and visceral organs. Several of these substances can induce both an acute and chronic inflammatory response (Sandkühler 2009, Boyce-Rustay et al 2010). Moreover, they have been backwardly validated by the effectiveness of opioids and non-anti-inflammatory drugs in controlling pain induced after their administration (Ren and Dubner 1999, Radhakrishnan et al 2003, Wilson et al 2006, Boyce-Rustay et al 2010, Gregory et al 2013).

Formalin ( 0.5 to $5 \%$ formaldehyde) injection induces inflammation and pain, with two temporal phases of different duration and underlying mechanisms (Tjølsen et al 1992). The acute phase (Phase I) is short lasting, mainly mediated by activation of the transient receptor potential cation channel (TRPV) (McNamara et al 2007). After a brief quiescent period, the acute phase is followed by a continuous and longer lasting phase (Phase II) that is supposedly mediated by instauration of a central sensitization state due to the continuous nociceptive transmission from type $\mathrm{C}$ fibres. Phase I of formalin test can be attenuated by local anaesthetics, while morphine, NMDA antagonist and gabapentin inhibit Phase II, but not Phase I (Dubuisson and Dennis 1977, Matthies and Franklin 1992, Abbadie et al 1997, McNamara et al 2007). Carrageenan is a water-extractable polysaccharide derived from the marine plants Gigartina aciculaire and Gigartina pistillata. Carrageenan injection promotes the instauration of an acute inflammatory
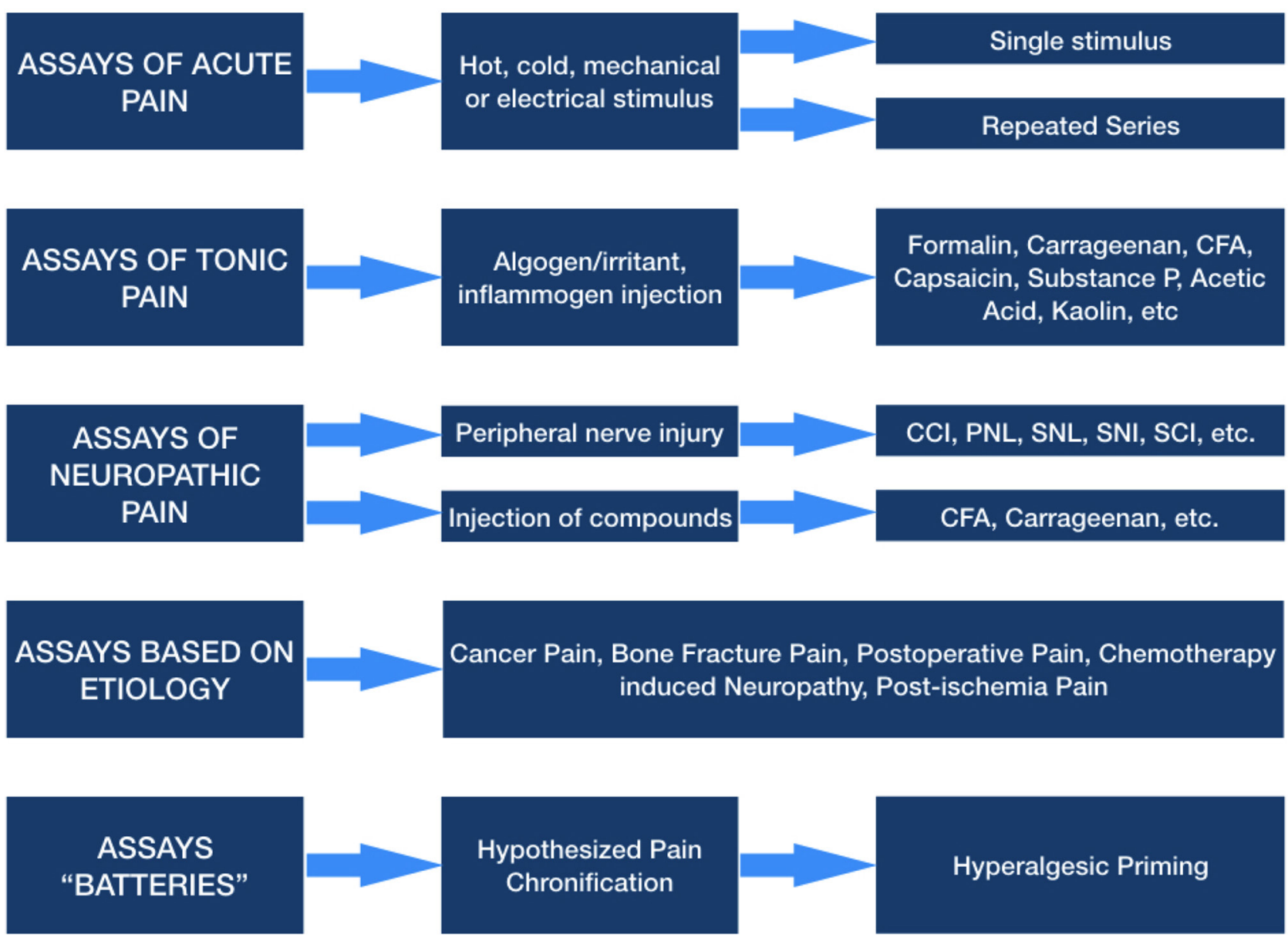

CFA: Complete Freund's Adjuvant; CCI: Chronic Constriction Injury; PNL: Partial Nerve Ligation; SNL: Spinal Nerve Ligation; SNI: Spinal Nerve Injury; SCI; Spinal Cord Injury.

Figure 1. Summary of current assays used in animal models of pain (Summarised from Mogil (2018). 
state that converts into chronic after approximately 2 weeks. Carrageenan can induce mechanical and thermal hyperalgesia in the injured site, as well as in the non-affected area, both mediated by peripheral and central sensitization, respectively (Radhakrishnan et al 2003). Carrageenan injection can also enhance avoidance, spontaneous and guarding pain behaviours as well as a reduction in the weight bearing force of an affected limb (Radhakrishnan et al 2004, Gregory et al 2013). Complete Freund's adjuvant (CFA) is an emulsion of heat-killed bacteria that includes Mycobacterium butyricum or Mycobacterium tuberculosis in sterile mineral oil (Swingle and Grant 1977). CFA injection induces an immune-mediated chronic inflammation of the surrounding tissue. This chronic inflammation is longer than that induced by carrageenan, nonetheless, clinical features mechanical and thermal primary and secondary hyperalgesia are well identified after CFA administration. Moreover, CFA injection modifies the behaviour of animals during the conditioned place preference (CPP) and conditioned place avoidance (CPA) tests. CFA injection has been also used to model chronic inflammatory joint diseases such as in rheumatoid arthritis (Shippenberg et al 1988, Ren and Dubner 1999 Gregory et al 2013, Parvathy and Masocha 2013).

\section{ASSAYS OF NEUROPATHIC PAIN}

Neuropathic pain (NP) often remains as one of the most challenging conditions to treat (Colloca et al 2017). The traditional translational approach has consisted of multiple attempts to understand mechanisms in animal models and then apply these data into the clinic (Bouhassira and Attal 2016). Neuropathic pain models have been developed to mimic painful pathological conditions such as nerve trauma, nerve compression, low back pain or diabetic neuropathy (Mogil 2009). However, animal models for nociplastic conditions such as fibromyalgia have not been developed (Yezierski and Hansson 2018). Peripheral nervous system (PNS) damage and its associated pain have been mainly modelled using the surgical intervention of a peripheral nerve, with the most common being the sciatic nerve and its branches (Colleoni and Sacerdote 2010, Gregory et al 2013, Challa 2015). Several animal models of NP using mechanical peripheral nerve injury to induce changes such as allodynia and hyperalgesia are currently described (Dowdall et al 2005). The most used models are nerve transection (NT) (Wall et al 1979), chronic constriction injury (CCI) (Bennett and Xie 1988), partial sciatic ligation (PNL) (Seltzer et al 1990), spinal nerve ligation (SNL)(Kim and Chung 1992), spare nerve injury of the tibial and peroneal nerve (SNI) (Decosterd and Woolf 2000), and inflammation (Maves et al 1993). All these different models are well characterised and all of them have advantages and disadvantages as well as limited ability to precisely model the clinical condition.
Nevertheless, these models have provided the basis for the mechanistic understanding that we have achieved to date on NP syndromes (Ossipov et al 2006).

\section{THE MEASURED OUTCOME}

Bedside pain models recreate a previously determined painful condition, nonetheless, they may also define a measurable and clinically translatable indicator (Klinck et al 2017). One of the most criticised aspects in pain research in the low capacity to identify and clinically use a specific, measurable, easy to score and translatable outcome (Blackburn-Munro 2004). Outcome measures are designed to evaluate multiple parts of the pain experience and can be broadly categorised in evoked and non-evoked measures.

Evoked responses during pain evaluate the behavioural response after heat, cold, mechanical, or electrical stimulation. Most of these behaviours are associated to spinal reflexes (i.e. limb withdrawal), spino-bulbospinal reflexes (i.e. jumping and abdominal stretching) and innate behaviour (vocalisation, licking, scratching, biting, guarding) and can also be observed in decerebrated animals (Matthies and Franklin 1992). Evoked measures are easy to perform and provide a quantifiable outcome. Interestingly, they have become the most frequent type of outcome reported in pain studies (Mogil 2009). Currently, an increasing debate about the capacity of this outcome to score the whole context of pain experience is underway (Vierck et al 2008). First, evoked pain reflexes have been useful to investigate the underlying mechanisms of pain hypersensitivity (hyperalgesia and/or allodynia), but not pain itself (Clark 2016). Secondly, evoked measures do not consider the cognitive and emotional aspect of pain (Mogil 2009, Quessy 2010); and thirdly, evoked tests are incapable to measure spontaneous pain states, which is by far the most frequent painful sensation reported by humans patients (Backonja and Stacey 2004). Furthermore, allodynia in human patients is frequently triggered by a dynamic component which differs from the mechanical allodynia evaluated using static pressure von Fray filaments in the affected tissue of rodents (Samuelsson et al 2005).

Non-evoked (operant) measures evaluate animal behaviours that require a functional spinal-cerebrospinal integration (Mogil 2009). Operant conditioning is based on the psychological theory in which learning occurs when a response to a stimulus is reinforced (learning theory) (Mogil 2018). If a positive reinforcement follows the response to a specific stimulus, then the response becomes more probable to occur thereafter. If a negative reinforcement occurs, or a reward is removed, the response becomes less probable. In this case, individuals may perform behaviours that terminate the exposure to the painful stimulus or increase the administration of analgesics (Mogil 2018). Positive reinforcement has been criticised by some researchers, mainly because if the pain response of the individuals is systematically reinforced in an early stage, it could 
be possible that the pain behaviour would continue after the original stimulus is terminated (Fordyce et al 1973).

Some studies using operant measures of pain behaviour have used paradigms such as condition place preference (CPP) and condition place avoidance (CPA) (Sufka 1994; Ding et al 2005). During CPP testing, a treatment group is allowed to gain access to an analgesic compound during the evaluation period and accordingly, the time spent in the analgesic administering chamber would indicate a preference. In contrast, during a CPA test, individuals are subsequently evaluated by an observer in order to discriminate the preference for a chamber in were no nociceptive stimulation occurs (Gregory et al 2013). These results could be used as indicators of aversiveness, which is clinically relevant because people with chronic pain markedly avoid painful stimulus (Mogil 2009).

Non-evoked measures have continuously demonstrated to be more concordant with clinical results than evoked measures. NP models evaluated using non-evoked measures have demonstrated a clear hypersensitivity for cold stimulation and not by heat, which is consistent with the clinical impression that cold allodynia is more common than heat allodynia after mechanical nerve damage. In contrast, evoked measures constantly demonstrate the opposite, with a notorious hypersensitivity to heat (Vierck et al 2005, Leffler and Hansson 2008).

An important limitation of these non-evoked paradigms is that they require significant learning with extensive training ( $\mathrm{Li}$ 2013). Nonetheless, fast learning procedures have been implemented, allowing to perform CCP testing after a 3-day pre-conditioning habituation (Okun et al 2011). Similarly, in non-evoked measurement, one motivating factor could be associated with behavioural response (Mogil 2009). Additionally, some authors have criticised that analgesic compounds could also play a role affecting the mental state of a painful animal which can then modify an operant behaviour (Gregory 2013).

One of the most common features of chronic pain is the development of spontaneous pain. Several behaviours have been evaluated for identifying spontaneous pain, including aggression, bite force, food intake, locomotion activity, rearing, struggling, weight bearing, posture and gait alteration (Mogil and Crager 2004). Similarly, other studies have focused on the use of more sophisticated techniques such as ultrasonic vocalisation and facial grimace scale (Han et al 2005, Sotocina et al 2011). Nonetheless, evaluating chronic pain using only behavioural indicators has proven complicated. Rodents do not manifest pain in a consistent way that could allow an evaluator to differentiate between mild and severe pain (Roughan and Flecknell 2003). Many of the proposed behaviours are not specific, some of them are affected by pain but do not represent ongoing pain. In some cases, the frequency of presentation of the behaviour is not frequent enough or highly variable among individuals (Graham and Hampshire 2016). Similarly, electroencephalography, functional magnetic resonance imaging (fMRI) and positron emission tomography (PET) have gathered great attention in the last couple of years regarding its ability to identify specific areas of the brain that become activated during a painful experience.

A different approach to obtain outcome measures is the use of methods of quantitative sensory testing (QST), which is a diagnostic tool that allows determining the sensitive perception evoked in response to a defined sensory stimulus. QST has been extensively used in human and rodents. The sensory modalities include small fibre sensory function, such as thermal detection/pain threshold and pinprick sensitivity, and large fibre sensory function, such as mechanical and vibration detection thresholds (Themistocleous et al 2018).

In summary, animal pain testing faces at least six main criticisms (Mogil et al 2010). These criticism include: a) the exaggerated emphasis on endpoints as withdrawal reflexes as dependent measurement; $b$ ) the lack of evaluating states that accompany chronic pain as sequelae or other comorbidities; c) the fact that most models are highly artificial including the administration of inflammatory mediators and surgical nerve damage; d) most primary symptoms of human chronic pain are spontaneous; e) the mismatch in the epidemiological prevalence of chronic pain in the human population and the usual choices of animal models and $\mathrm{f}$ ) differences in the standard design between animal experiments and human clinical trials (Vierck et al 2008, Vierck et al 2002, Brennan et al 1996, Scholz et al 2009, Rice et al 2008).

\section{NATURALLY OCCURRING PAIN MODELS}

Naturally occurring diseases and painful conditions that affect animals might better reflect the complex genetic, environmental and physiological variation present in humans (Kol et al 2015). Moreover, previous reports have strongly suggested the use of veterinary conditions as translational pain models (Lascelles et al 2018). A letter to the editor by Quessy (2010) in the prestigious The Journal of Pain mentioned at that time the urgency for a translational research agenda with a focus on natural diseases and validated, relevant outcome measures, suggesting that clinical trials in animals with natural disease may improve the predictive veracity for drug candidate selection, praising the role of Veterinary Medicine. Even more, Kol et al (2015) proposed that companion animals would play an important role in defining translational medicine, suggesting the need for identifying naturally occurring diseases with potential for accelerating translation. Moreover, Kol et al (2015) and Lascelles (2018) go one step forward and suggest a new paradigm in drug testing and clinical trials, incorporating a II phase veterinary clinical trial between the preclinical research and human clinical trials. Nonetheless, this intermediate step would require an important change in the form in which traditional pain research has been performed over the years. These changes 
would include experimental design (number of animals, replicability, reproducibility, among others) and ethical concerns that would limit large-scale studies.

Interestingly, in the last 5-10 years, Veterinary Medicine has stepped forward and have responded to the challenge, identifying and better defining naturally occurring conditions with potential translation, including the field of pain. Painful conditions commonly occur in veterinary patients including dogs and cats, horses, cattle, sheep, and swine. In most animals, these painful conditions develop naturally and usually over a long period of time, which better resembles the painful condition in human patients with chronic pain. A different asset to using translational pain models that occur naturally is the phylogenetical proximity between some of these species and humans (Obrien et al 2008, Hoeppner et al 2014). Lascelles et al (2018) mention that naturally occurring models may better reflect the complex genetic, environmental, temporal and physiological influences present in humans.

Some examples of painful conditions include tail docking (amputation), dehorning, castration, and lameness in cattle and sheep; osteoarthritis, denervation, chronic ligament injuries, and lumbar pain in horses; osteochondrosis, tail docking, castration in pigs; amputation, osteoarthritis, osteosarcoma, carcinoma, intervertebral disk disease, degenerative lumbosacral stenosis in dogs, and diabetic neuropathy, inflammatory mammary carcinoma, osteoarthritis, amputation, interstitial cystitis and inflammatory bowel disease in cats (Klink et al 2017).

Nonetheless, scientific evidence discussing how these conditions could represent a translational approach has only been reported for feline interstitial cystitis (Buffington 2001), feline diabetes mellitus (Mizisin et al 2002), osteoarthritis (McCoy 2015) and various tumours in dogs (Peña et al 2003, Brown et al 2009) and cats (Pérez-Alenza et al 2004), lameness in dairy cattle and horses (Bustamante et al 2015, Meneses et al 2018, Rodriguez et al 2018, Herzberg et al 2019), tail docking in dairy cows (Troncoso et al 2018). According to Klinck et al (2017), this relatively limited evidence could be indicative of the complexity in the design and interpretation of studies in which the number of recruited subjects is reduced. Additionally, as in experimental animal models, limitation exist in naturally occurring conditions, which confirms that predictive results should be analysed with caution. Nonetheless, we strongly believe these limitations represent an opportunity to develop novel research strategies, including the confirmation of previously described pathophysiological mechanisms using a genomic and proteomic approach.

Interestingly, the vast majority of the test used in experimental models have been evaluated in naturally occurring models, including thermal and mechanical thresholds, activity monitoring, operant testing, fMRI, PET and electroencephalography. Troncoso et al (2018) used a QST battery in order to confirm the presence of chronic pain in long-term tail docked dairy cows. Probably the most studied and well-defined translational model of chronic pain is companion animals' osteoarthritis. Lascelles et al (2018) confirmed that spontaneous painful osteoarthritis in companion animals offers translational potential.

Recently, Lascelles et al (2019) discussed the priorities and future research for measurement of chronic pain in companion animals. This interesting review concludes that improvements must be made in the development and standardisation of clinical metrology instruments, including partnering with owners; in better defining and evaluating health-related quality of life (HRQoL), in the measurement of animals that suffer chronic pain; advance in computational gait analysis instrumentation; further studies in QST methodology; advance in the definition of nociceptive withdrawal reflex in companion animals; obtain outcome measures for cancer pain and define the effect of placebo on outcome measures. Moreover, preliminary findings should be taken with caution, considering that several of the concerns represented in traditional pain models could also be present in naturally occurring painful conditions. These considerations led to probably the most important conclusion: collaboration is the path forward in chronic pain diagnosing and management in both human and veterinary medicine.

Finally, here we have described the current limitations of animal models of chronic pain and briefly explained how naturally occurring pain models could represent a translational approach. We consider that Veterinary Medicine must play an important role in the finding, development, confirmation and applicability of potential translational models that would help solve human and non-human animals' medical conditions under the concepts of one health, one welfare and one pain.

\section{REFERENCES}

Abbadie C, Taylor BK, Peterson MA, Basbaum AI. 1997. Differential contribution of the two phases of the formalin test to the pattern of c-fos expression in the rat spinal cord: studies with remifentanil and lidocaine. Pain 69, 101-110.

Adrian D, Papich M, Baynes R., Murrell J, Lascelles, BD. 2017. Chronic maladaptative pain in cats: A review of current and future drug treatment options. Vet J 230, 52-61.

Arrowsmith J. 2012. A decade of change. Nat Rev Drug Discov 11, 17-18.

Backonja MM, Stacey B. 2004. Neuropathic pain symptoms relative to overall pain rating. J Pain 5, 491-497.

Bennett GJ, Xie YK. 1988. A peripheral mononeuropathy in rat that produces disorders of pain sensation like those seen in man. Pain 33, 87-107.

Blackburn-Munro G. 2004. Pain-like behaviors in animals - how human are they?. Trends Pharmacol Sci 25, 299-305.

Bouhassira D, Attal N. 2016. Translational neuropathic pain research: a clinical perspective. Neuroscience 338, 27-35.

Boyce-Rustay JM, Honore P, Jarvis MF. 2010. Animal models of acute and chronic inflammatory and nociceptive pain. Methods Mol Biol 617, 41-55.

Brennan TJ, Vandermeulen EP, Gebhart GF. 1996. Characterization of a rat model of incisional pain. Pain 64, 493-502. 
Brown DC, Boston R, Coyne JC, Farrar JT. 2009. A novel approach to the use of animals in studies of pain: validation of the canine brief pain inventory in canine bone cancer. Pain Med 10, 133-142.

Buffington CA. 2001. Visceral pain in humans: lessons from animals. Curr Pain Headache Rep 5, 44-51.

Burma NE, Leduc-Pessah H, Fan CY, Trang T. 2017. Animal models of chronic pain: Advances and challenges for clinical translation. $J$ Neurosci Res 95, 1242-1256.

Bustamante HA, Rodriguez AR, Herzberg DE, Werner MP. 2015. Stress and pain response after oligofructose induced-lameness in dairy heifers. J Vet Sci 16, 405-411.

Challa SR. 2015. Surgical animal models of neuropathic pain: pros and cons. Int J Neurosci 125, 70-174.

Clark JD. 2016. Preclinical Pain Research: Can We Do Better?. Anesthesiology 125, 846-849.

Colleoni M, Sacerdote P. 2010. Murine models of human neuropathic pain. Biochim Biophys Acta 1802, 924-933.

Colloca L, Ludman T, Bouhassira D, Baron R, Dickenson AH, et al. 2017. Neuropathic pain. Nat Rev Dis Primers 3, 17002.

Decosterd I, Woolf CJ. 2000. Spared nerve injury: an animal model of persistent peripheral neuropathic pain. Pain 87, 149-158.

Ding HK, Shum FWF, Ko SW, Zhuo M. 2005. A new assay of thermal-based avoidance test in freely moving mice. J Pain 6, 411-416.

Dowdall T, Robinson I, Meert T. 2005. Comparison of five different rat models of peripheral nerve injury. Pharmacol Biochem Be 80, 93-108.

Dubuisson D, Dennis SG. 1977. The formalin test: a quantitative study of the analgesic effects of morphine meperidine and brain stem stimulation in rats and cats. Pain 4, 161-174.

Enright A, Goucke R. 2016. The global burden of pain: the tip of the iceberg?. Anesth Analg 123, 529-530.

Fordyce WE, Fowler Jr RS, Lehmann JF, Delateur BJ, Sand PL, et al. 1973. Operant conditioning in the treatment of chronic pain. Arch Phys Med Rehabil 54, 399-408.

Gereau IV RW, Sluka KA, Maixner W, Savage SR, Price TJ, et al. 2014. A pain research agenda for the $21^{\text {st }}$ century. $J$ Pain $15,1203-1214$.

Graham DM, Hampshire V. 2016. Methods for measuring pain in laboratory animals. Lab Anim 45, 99-101.

Gregory NS, Harris AL, Robinson CR, Dougherty PM, Fuchs PN, et al. 2013. An overview of animal models of pain: disease models and outcome measures. J Pain 14, 1255-1269.

Han JS, Bird GC, Li W, Jones J, Neugebauer V. 2005. Computerized analysis of audible and ultrasonic vocalizations of rats as a standardized measure of pain-related behavior. J Neurosci Methods 141, 261-269.

Hay M, Thomas DW, Craighead JL, Economides C, Rosenthal J. 2014. Clinical development success rates for investigational drugs. Nat Biotechnol 32, 40-51.

Herzberg D, Strobel P, Chihuailaf R, Ramirez-Reveco A, Müller H, et al. 2019. Spinal reactive oxygen species and oxidative damage mediate chronic pain in lame dairy cows. Animals $9,963$.

Hoeppner MP, Lundquist A, Pirun M, Meadows JR, Zamani N, et al. 2014. An improved canine genome and a comprehensive catalogue of coding genes and non-coding transcripts. PloS One 9, e91172.

Jackson T, Thomas S, Stabile V, Shotwell M, Han X, et al. 2016. A systematic review and meta-analysis of the global burden of chronic pain without clear etiology in low- and middle-income countries: trends in heterogeneous data and a proposal for new assessment methods. Anesth Analg 123, 739-748.

Kim SH, Chung JM. 1992. An experimental-model for peripheral neuropathy produced by segmental spinal nerve ligation in the rat. Pain 50, 355-363.

Klinck MP, Mogil JS, Moreau M, Lascelles BDX, Flecknell PA, et al. 2017. Translational pain assessment: could natural animal models be the missing link?. Pain 158, 1633-1646.

Kol A, Boaz A, Athanasiou K, Farmer DL, Nolta JA, et al. 2015. Companion animals: Translational scientist's new best friends. Sci Transl Med 7, 308ps21308ps21.
Kumar K, Elavarasi P. 2016. Definition of pain and classification of pain disorders. J Adv Clin Res Ins 3, 87-90.

Lascelles BDX, Brown DC, Maixner W, Mogil JA. 2018. Spontaneous painful disease in companion animals can facilitate the development of chronic pain therapies for humans. Osteoarthr Cartilage 26, 175-183.

Lascelles BDX, Brown DC, Conzemius M, Gill M, Oshinsky ML, et al. 2019. Measurement of chronic pain in companion animals: Priorities for future research and development based on discussion from the Pain in Animals Workshop (PAW) 2017. Vet J 252, 105370.

Leffler AS, Hansson P. 2008. Painful traumatic peripheral partial nerve injury-sensory dysfunction profiles comparing outcomes of bedside examination and quantitative sensory testing. Eur J Pain 12, 397-402.

Li JX. 2013. The application of conditioning paradigms in the measurement of pain. Eur J Pharmacol 716, 158-168.

Mao J. 2009. Translational pain research: achievements and challenges. J Pain 10, 1001-1011.

Matthies BK, Franklin KB. 1992. Formalin pain is expressed in decerebrate rats but not attenuated by morphine. Pain 51, 199-206.

Maves T, Pechman P, Gebhart G, Meller S. 1993. Possible chemical contribution from chromic gut sutures produces disorders of pain sensation like those seen in man. Pain 54, 57-69.

McCoy AM. 2015. Animal models of osteoarthritis: comparisons and key considerations. Vet Pathol 52, 803-818.

McNamara CR, Mandel-Brehm J, Bautista DM, Siemens J, Deranian KL, et al. 2007. TRPA1 mediates formalin-induced pain. Proc Natl Acad Sci USA 104, 13525-13530.

Meneses CS, Müller HY, Herzberg DE, Uberti B, Werner MP, et al. 2018. Microglia and astrocyte activation in the spinal cord of lame horses. Vet Anaesth Analg 45, 92-102.

Mizisin AP, Shelton GD, Burgers ML, Powell HC, Cuddon PA. Neuroligical complications associated with spontaneous occuring feline diabetes mellitus. J Neuropathol Exp Neurol 61, 872-884.

Mogil JS, Crager SE. 2004. What should we be measuring in behavioral studies of chronic pain in animals? Pain 112, 12-15.

Mogil JS. 2009. Animal models of pain: progress and challenges. Nat Rev Neurosci 10, 283-294.

Mogil JS, Davis KD, Derbyshire SW. 2010. The necessity of animal models in pain research. Pain 151, 12-17.

Mogil JS. 2012. The etiology and symptomatology of spontaneous pain. J Pain 13, 932-933.

Mogil JS. 2018. The Measurement of Pain in the Laboratory Rodent. Oxford University Press, Oxford, UK.

O'Brien SJ, Johnson W, Driscoll C, Pontius J, Pecon-Slattery J, et al. 2008. State of cat genomics. Trends Genet 24, 268-279.

Okun A, DeFelice M, Eyde N, Ren J, Mercado R, et al. 2011. Transient inflammation-induced ongoing pain is driven by TRPV1 sensitive afferents. Mol Pain 7, 4.

Ossipov MH, Lai J, Porreca F. 2006. Mechanisms of experimental neuropathic pain: integration from animal models. In: McMahon SB, Koltzenburg M (eds). Wall and Melzack's Textbook of Pain. Elsevier, Philadelphia, USA.

Parvathy SS, Masocha W. 2013. Gait analysis of C57BL/6 mice with complete Freund's adjuvant-induced arthritis using the CatWalk system. BMC Musculoskelet Disord 14, 14

Peña L, Perez-Alenza MD, Rodriguez-Bertos A, Nieto A. 2003. Canine inflammatory carcinoma: histopathology, immunohistochemistry and clinical implications of 21 cases. Breast Cancer Res Treat 78, 141-148.

Perez-Alenza MD, Jiménez A, Nieto AI, Peña L. 2004. First description of feline inflammatory mammary carcinmoa: clinicopathologican and immunohistochemical characteristics of three cases. Breast Cancer Res 6, R300-R307.

Quessy SN. 2010. The challenges of translational research for analgesics: the state of knowledge needs upgrading, and some uncomfortable deficiencies remain to be urgently addressed. J Pain 11, 698-700.

Radhakrishnan R, Moore SA, Sluka KA. 2003. Unilateral carrageenan injection into muscle or joint induces chronic bilateral hyperalgesia in rats. Pain 104, 567-577. 
Radhakrishnan R, Bement MKH, Skyba D, Sluka KA, Kehl LJ. 2004 Models of muscle pain: carrageenan model and acidic saline model. Curr Protoc Pharmacol 25, 5.35.1-5.35.28

Ren K, Dubner R. 1999. Inflammatory models of pain and hyperalgesia. ILAR J 40, 111-118.

Rice ASC, Cimino-Brown D, Eisenach JC, Kontinen VK, LaCroixFralish, et al. 2008. Animal models and the prediction of efficacy in clinical trials of analgesic drugs: a critical appraisal and call for uniform reporting standards. Pain 139, 241-245.

Rodriguez AR, Herzberg DE, Werner MP, Müller HY, Bustamante HA 2018. Plasma concentration of norepinephrine, beta-endorphin, and substance P in lame dairy cows. J Vet Res 62, 193-197.

Roughan JV, Flecknell PA. 2003. Evaluation of a short duration behavior-based post-operative pain scoring system in rats. Eur J Pain 7, 397-406.

Samuelsson MA, Leffler S, Hansson P. 2005. Dynamic mechanical allodynia: on the relationship between temporo-spatial stimulus parameters and evoked pain in patients with peripheral neuropathy. Pain 115, 264-272.

Sandkühler J. 2009. Models and mechanisms of hyperalgesia and allodynia. Physiol Rev 89, 707-758.

Scholz J, Mannion RJ, Hord DE, Griffin RS, Rawal B, et al. 2009. A novel tool for the assessment of pain: validation in low back pain. PloS Med 6:e1000047.

Seltzer Z, Dubner R, Shir Y. 1990. A novel behavioral-model of neuropathic pain disorders produced in rats by partial sciatic-nerve injury. Pain 43, 205-218.

Shippenberg TS, Stein C, Huber A, Millan MJ, Herz A. 1988. Motivational effects of opioids in an animal model of prolonged inflammatory pain: alteration in the effects of kappa- but not of mu-receptor agonists. Pain 35, 179-186.

Sotocinal SG, Sorge RE, Zaloum A, Tuttle AH, Martin LJ, et al. 2011. The rat grimace scale: a partially automated method for quantifying pain in the laboratory rat via facial expressions. Mol Pain 7, 55.

Sufka KJ. 1994. Conditioned place preference paradigm: a novel approach for analgesic drug assessment against chronic pain. Pain 58, 355-366.

Sunil kumar Reddy K, Naidu MUR, Usha Rani P, Ramesh Kumar Rao T. 2012. Human experimental pain models: A review of standardized methods in drug development. J Res Med Sci 17, 587-595.

Swingle KF, Grant TJ. 1977. Evaluation of some standard arthritogenic adjuvants ("modified perrigens") in male rats. Agents Actions 7, 459-463.
Themistocleous AC, Crombez G, Baskozos G, Bennett DL. 2018. Using stratified medicine to understand, diagnose, and treat neuropathic pain. Pain 159, S31-S42.

Tjølsen A, Berge OG, Hunskaar S, Rosland J, Hole K. 1992. The formalin test: an evaluation of the method. Pain 51, 5-17.

Treede RD, Rief W, Barke A, Aziz Q, Bennett MI, et al. 2019. Chronic pain as a symptom or a disease: the IASP classification of chronic pain for the International Classification of Diseases (ICD-11). Pain 160, 19-27.

Treede RD, Rief W, Barke A, Aziz Q, Bennett MI, et al. 2015. A classification of chronic pain for ICD.11. Pain 156, 1003-1007.

Troncoso RJ, Herzberg DE, Meneses CS, Müller HY, Werner MP, et al. 2018. Mechanical/thermal sensitivity and superficial temperature in the stump of long-term tail-docked dairy cows. PeerJ 18, 6:e5213.

Ueda H. 2008. Peripheral mechanisms of neuropathic pain - involvement of lysophosphatidic acid receptor-mediated demyelination. Mol Pain 1, 4-11.

Vierck CJ, Acosta-Rua AJ, Nelligan R, Tester N, Mauderli A. 2002. Low dose systemic morphine attenuates operant escape but facilitates innate reflex responses to thermal stimulation. J Pain 3, 309-319.

Vierck CJ, Acosta-Rua AJ, Johnson RD. 2005. Bilateral chronic constriction of the sciatic nerve: a model of long-term cold hyperalgesia. J Pain 6, 507-517.

Vierck CJ, Hansson PT, Yezierski RP. 2008. Clinical and pre-clinical pain assessment: are we measuring the same thing?. Pain 135, 7-10.

Wall P, Scadding JW, Tomkiewicz MM. 1979. The production and prevention of experimental anesthesia dolorosa. Pain 6, 175-182.

Walker K, Fox AJ, Urban LA. 1999. Animal models for pain research. Molecular Medicine Today 5, 319-321.

Weyer AD, Zappia KJ, Garrison SR, O'Hara CL, Dodge AK. 2016. Nociceptor sensitization depends on age and pain chronicity. eNeuro 3, 1-26.

Wilson SG, Mogil JS. 2001. Measuring pain in the (knockout) mouse: big challenges in a small mammal. Behav Brain Res 125, 65-73.

Wilson AW, Medhurst SJ, Dixon I, Bontoft NC, Winyard LA, et al. 2006. An animal model of chronic inflammatory pain: pharmacological and temporal differentiation from acute models. Eur J Pain 10, 537-549.

Woolf CJ. 2010. What is this thing called pain?. J Clin Invest 120, 3742-3744.

Woolf CJ. 2011. Central sensitization: implications for the diagnosis and treatment of pain. Pain 152, S2-S15.

Yezierski RP, Hansson P. 2018. Inflammatory and neuropathic pain from bench to bedside: what went wrong?. J Pain 19, 571-588. 